\title{
SAMUEL SELLAM
}

\section{ALAIN DUSSAUCHOY}

\section{Une mesure pour l'interprétation d'un ensemble de facteurs dans une analyse en composantes principales}

Revue française d'automatique, d'informatique et de recherche opérationnelle. Recherche opérationnelle, tome 14, n 1 (1980), p. 43-51.

<http://www.numdam.org/item?id=RO_1980_14_1_43_0>

C AFCET, 1980, tous droits réservés.

L'accès aux archives de la revue « Revue française d'automatique, d'informatique et de recherche opérationnelle. Recherche opérationnelle » implique l'accord avec les conditions générales d'utilisation (http://www.numdam.org/ legal.php). Toute utilisation commerciale ou impression systématique est constitutive d'une infraction pénale. Toute copie ou impression de ce fichier doit contenir la présente mention de copyright.

\section{Numdam}

Article numérisé dans le cadre du programme

Numérisation de documents anciens mathématiques

http://www.numdam.org/ 


\title{
UNE MESURE POUR L'INTERPRÉTATION D'UN ENSEMBLE DE FACTEURS DANS UNE ANALYSE EN COMPOSANTES PRINCIPALES $\left({ }^{*}\right)$
}

\author{
par Samuel Sellam $\left({ }^{1}\right)$ et Alain Dussauchoy $\left({ }^{1}\right)$
}

\begin{abstract}
Résumé. - On présente une généralisation de la notion de « contribution d'un facteur à la variance d'une variable » [1] au cas d'un ensemble de facteurs par rapport à un ensemble de variables et l'on démontre ensuite que cette contribution peut être exprimée en fonction $d u$ "Produit scalaire entre opérateurs » $[4,5,3]$ d'Escoufier.

Abstract. - Here, the notion of "factor contribution to the variance of a variable" is generalized to the case of several factors with respect to several variables. It is shown that this contribution can be expressed in terms of the "scalar product between operators" (Escoufier [4, 5, 3]).
\end{abstract}

\section{RAPPEL DE LA NOTION DE « CONTRIBUTION D'UN FACTEUR A LA VARIANCE D'UNE VARIABLE "}

Soit $\mathscr{X}$ un vecteur aléatoire à $n$ dimensions constitué des $n$ variables aléatoires centrées à analyser.

Soit $V$ la matrice de variance-covariance du vecteur aléatoire $\mathscr{X}$.

Soit $D_{\lambda}$ la matrice diagonale des valeurs propres de $V$ et $C$ sa matrice des vecteurs propres normalisés.

Soit $\mathscr{Y}$ le vecteur aléatoire des facteurs défini à partir du vecteur aléatoire $\mathscr{X}$ par l'équation

$$
\mathscr{Y}=C^{t} \mathscr{X}
$$

La propriété d'orthogonalité $\left({ }^{2}\right)$ de la matrice $C$ permet d'écrire (1) sous la forme

$$
\mathscr{X}=C \mathscr{Y} .
$$

\footnotetext{
$\left({ }^{*}\right)$ Reçu janvier 1979.

(1) Laboratoire des Méthodes informatiques appliquées à la Gestion, Université Claude-Bernard, Lyon-I.

$\left({ }^{2}\right) C^{t} C=C C^{t}=I_{n}$ matrice unité.
}

R.A.I.R.O. Recherche opérationnelle/Operations Research, 0399-0842/1980/43/S 5.00

(C) AFCET-Bordas-Dunod. 
Soit $X_{p}$ la $p$-ième variable aléatoire de $\mathscr{X}$, si on note $c_{\text {. }}$ la $p$-ième ligne de $C$, on peut déduire de (2) l'expression de $X_{p}$ en fonction de $\mathscr{Y}$ :

$$
X_{p}=c_{. p} \mathscr{Y}
$$

Considérons maintenant la variance de la variable aléatoire $X_{p}$ :

$$
\operatorname{Var}\left(X_{p}\right)=E\left[X_{p} X_{p}^{t}\right]
$$

Du fait de (3) et de la linéarité de l'espérance mathématique on en déduit

$$
\operatorname{Var}\left(X_{p}\right)=E\left[c_{\cdot p} \mathscr{Y} \mathscr{Y}^{t} c_{\cdot p}^{t}\right]=c_{\cdot p} E\left[\mathscr{Y} \mathscr{Y}^{t}\right] c_{\cdot p}^{t} .
$$

Or, les variables aléatoires $Y_{j}$ du vecteur $\mathscr{Y}$ sont non corrélées par construction donc

$$
E\left[\mathscr{y} \mathscr{Y}^{t}\right]=D_{\lambda}
$$

[Le j-ième élément de $D_{\lambda}$ étant la variance de $\left.Y_{j}\left(\operatorname{Var}\left(Y_{j}\right)\right)\right]$.

On peut donc écrire (4) sous la forme

$$
\operatorname{Var}\left(X_{p}\right)=\sum_{j=1}^{n} c_{j p}^{2} \operatorname{Var}\left(Y_{j}\right)
$$

L'équation (6) exprime la linéarité de la variance des variables en fonction des variances des facteurs. On peut donc en déduire que la contribution brute du facteur $Y_{l}$ à la variance de $X_{p}$ est la quantité $c_{l p}^{2}$ var $\left(Y_{l}\right)$ et sa contribution relative notée $\mathscr{C}_{Y, / X_{p}}$ sera donnée par

$$
\mathscr{C}_{Y_{l} / X_{p}}=\frac{c_{l p}^{2} \operatorname{Var}\left(Y_{l}\right)}{\sum_{j=1}^{n} c_{j p}^{2} \operatorname{Var}\left(Y_{j}\right)}=\frac{c_{l p}^{2} \operatorname{Var}\left(Y_{l}\right)}{\operatorname{Var}\left(X_{p}\right)}
$$

\section{II. “ CONTRIBUTION D'UN ENSEMBLE DE FACTEURS A LA VARIANCE D'UN ENSEMBLE DE VARIABLES "}

Soit $\mathscr{X}_{l}$ un vecteur aléatoire formé de $l$ variables aléatoires du vecteur $\mathscr{X}$ et, soit $\mathscr{Y}_{k}$ un vecteur aléatoire formé de $k$ facteurs du vecteur $\mathscr{Y}$.

Si on définit la quantité $\mathscr{I}_{\mathscr{X}_{\imath}}$ comme :

$$
\mathscr{I}_{X_{l}}=\sum_{j=1}^{l} \operatorname{Var}\left(X_{j}\right)
$$

( $\mathscr{I}$ n'est autre qu'une généralisation de la variance [3]).

R.A.I.R.O. Recherche opérationnelle/Operations Research 
Compte tenu de l'équation (3), on peut écrire :

$$
\mathscr{I}_{\mathscr{X}_{t}}=\sum_{j=1}^{l} E\left[X_{j} X_{j}^{t}\right]=\sum_{j=1}^{l} E\left[c_{. j} \mathscr{Y Y Y}^{t} c_{. j}^{t}\right] .
$$

Comme pour (4) la linéarité de l'espérance mathématique permet d'écrire :

$$
\mathscr{I}_{\mathscr{X}_{1}}=\sum_{j=1}^{l} c_{. j} E\left[\mathscr{Y O Y}{ }^{t}\right] c_{. j}^{t} .
$$

Soit, compte tenu de (5) :

$$
\mathscr{I}_{x_{i}}=\sum_{j=1}^{l} \sum_{i=1}^{n} c_{i j}^{2} \operatorname{var}\left(Y_{i}\right)
$$

Si on regroupe les $k$ facteurs de $\mathscr{Y}_{k}$ on a

$$
\mathscr{I}_{X_{i}}=\sum_{i=1}^{k} \sum_{j=1}^{l} c_{i j}^{2} \operatorname{Var}\left(Y_{i}\right)+\sum_{i=k+1}^{n} \sum_{j=1}^{l} c_{i j}^{2} \operatorname{Var}\left(Y_{i}\right) \text {. }
$$

Ainsi $\mathscr{I}_{\mathscr{X}_{1}}$ est réparti entre une contribution de $\mathscr{Y}_{k}$ et une contribution du complémentaire dans $\mathscr{Y}$ de $\mathscr{Y}_{k}$ (noté dans la suite $\mathscr{Y}_{k}^{\perp}$ ).

On peut donc en déduire l'expression de la « contribution de $k$ facteurs à la variance de $l$ variables $»$ que l'on notera $\mathscr{C}_{\mathscr{O}_{k} / \mathscr{X}_{l}}$ :

$$
\mathscr{C}_{\mathscr{X}_{k} / \mathscr{X}_{i}}=\frac{\sum_{i=1}^{k} \sum_{j=1}^{l} c_{i j}^{2} \operatorname{Var}\left(Y_{i}\right)}{\sum_{j=1}^{l} \sum_{i=1}^{n} c_{i j}^{2} \operatorname{Var}\left(Y_{i}\right)}=\frac{\sum_{i=1}^{k} \sum_{j=1}^{l} c_{i j}^{2} \operatorname{Var}\left(Y_{i}\right)}{\sum_{j=1}^{l} \operatorname{Var}\left(X_{j}\right)} .
$$

Une autre expression de $\mathscr{C}_{y_{\alpha} / \mathscr{X}_{4}}$ plus intéressante pour la suite peut être établie : pour cela il faut introduire les éléments suivants :

Soit $B$ la sous-matrice $(n \times l)$ composée des $l$ colonnes de la matrice $C^{t}$ correspondant aux $l$ variables de $\mathscr{X}_{l}$ et $A$ la sous-matrice $(k \times l)$ composée des $k$ lignes de $B$ correspondant aux $k$ facteurs de $\mathscr{Y}_{k}$.

Et sil'on convient que $D_{\lambda k}$ est la matrice $(k \times k)$ diagonale des valeurs propres de $\mathscr{Y}_{k}$, alors on pourra vérifier que :

$$
\mathscr{C}_{\mathscr{J}_{k} / X_{t}}=\frac{\operatorname{Trace}\left(A^{t} D_{\lambda k} A\right)}{\operatorname{Trace}\left(B^{t} D_{\lambda} B\right)} .
$$

\section{PROPRIÉTÉS DE LA « CONTRIBUTION » $\mathscr{C}$}

Le lecteur pourra aisément vérifier les trois propriétés suivantes :

$$
\begin{aligned}
& \mathrm{III}_{1}=\operatorname{Si} k=n \text { alors } \mathscr{C}_{\mathscr{y}_{k} / \mathscr{X}_{i}}=1 \text { pour tout } \mathscr{X}_{l} ; \\
& \mathrm{III}_{2}=\mathrm{Si} l=n \text { alors } \mathscr{C}_{y_{k} / \mathscr{X}_{i}}=\sum_{j=1}^{k} \lambda_{j} / \sum_{j=1}^{n} \lambda_{j} ;
\end{aligned}
$$


Avec $\lambda_{j}$ la $j$-ième valeur propre :

Nous reconnaissons en III $_{2}$ la formule de la part de variance expliquée par $k$ facteurs :

$\mathrm{III}_{3}=\mathscr{C}_{\mathscr{Y}_{k} / \mathscr{X}_{t}}+\mathscr{C}_{y_{i} \perp / X_{!}}=1$.

On dispose ainsi d'une mesure pour interpréter n'importe quel sous-ensemble de facteurs déduits d'une analyse en composantes principales et nous allons voir que cette mesure, si elle ne répond pas aux axiomes de la distance, peut toutefois s'exprimer en fonction du produit scalaire entre opérateurs d'Escoufier.

\section{RAPPEL DE LA MISE EN CEUVRE DU PRODUIT SCALAIRE ENTRE OPÉRATEURS $[3,5]$}

Soit le tableau $X$ des $p$ caractères notés $x^{i}$ mesurés sur le nuage $I$ des $n$ individus muni de la métrique $M_{1}$ et, soit le tableau $Y$ des $q$ caractères notés $y^{j}$ mesurés sur le même nuage $I$ muni de la métrique $M_{2}$.

On a le double schéma de dualité suivant: (pour plus de détails sur le formalisme employé voir [3] ou [5]) :

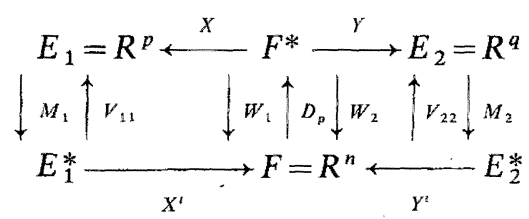

Avec $F^{*}, E_{1}^{*}, E_{2}^{*}$ les espaces duaux de $F$ (espace des caractères), $E_{1}$ (espace des individus repérés par les $p$ caractères $x^{i}$ ), $E_{2}$ (espace des individus repérés par les $q$ caractères $Y^{j}$ ) respectivement et :

- $D_{p}$ la métrique diagonale des poids des $n$ individus dans l'espace des caractères;

$-V_{11}=X D_{p} X^{t}, V_{12}=X D_{p} Y^{t}=V_{21}^{t}, V_{22}=Y D_{p} Y^{t}$

- $W_{1}=X^{t} M_{1} X, U_{1}=W_{1} D_{p}, W_{2}=Y^{t} M_{2} Y, U_{2}=W_{2} D_{p}$.

La comparaison des triplets $\left(X, M_{1}, D_{p}\right)$ et $\left(Y, M_{2}, D_{p}\right)$ revient à la comparaison des opérateurs $U_{1}$ et $U_{2}$.

Nous rappelons que le produit scalaire entre opérateurs d'Escoufier s'exprime par

$$
P\left(U_{1}, U_{2}\right)=\text { Trace }\left(U_{1} U_{2}\right)
$$

Si $U_{1}$ et $U_{2}$ sont les matrices associées aux opérateurs $U_{1}$ et $U_{2}$.

R.A.I.R.O. Recherche opérationnelle/Operations Research 
Nous rappelons également sans le démontrer un résultat qui nous sera utile

$$
P\left(U_{1}, U_{2}\right)=\operatorname{trace}\left(U_{1} U_{2}\right)=\operatorname{trace}\left(V_{21} M_{1} V_{12} M_{2}\right) .
$$

\section{LE SCHÉMA DE DUALITÉ D'UNE ANALYSE EN COMPOSANTES PRINCIPALES}

Soit le tableau $X$ de $p$ caractères $x^{i}$ mesurés sur le nuage $I$ des $n$ individus muni de la métrique $M_{1}$.

On rappelle qu'une analyse en composantes principales revient à calculer entre autres choses la matrice $C$ des vecteurs propres d'une certaine matrice notée $V_{11}$ des variances covariances des caractères.

Tenant compte de ce qui précède :

Soit le tableau $Y=C^{t} X\left({ }^{3}\right)$ des $p$ facteurs $y^{j}$ « mesurés » sur le nuage $I$ muni de la métrique $M_{2}$.

Nous aurons le schéma de dualité suivant :

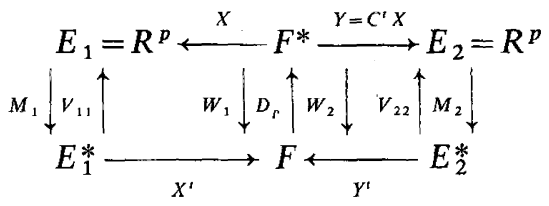

Notons que dans le cas d'une analyse en composantes principales $V_{22}$ n'est autre que $D_{\lambda}$ la matrice diagonale des valeurs propres donc, nous pourrons écrire en écriture matricielle

$$
\begin{gathered}
V_{11}=X D_{p} X^{t}=C D_{\lambda} C^{t} \\
V_{22}=Y D_{p} Y^{t}=C^{t} X D_{p} X^{t} C=C^{t} V_{11} C=D_{\lambda} \\
V_{12}=X D_{p} Y^{t}=X D_{p} X^{t} C=V_{11} C=C^{t} D_{\lambda} \\
V_{21}=C^{t} V_{11}=D_{\lambda} C^{t}
\end{gathered}
$$

\section{PRODUIT SCALAIRE ENTRE OPÉRATEURS DEFINIS SUR DES SOUS-ENSEMBLES DE CARACTÈRES}

Restant dans le cadre d'une analyse en composantes principales, nous allons procéder à une projection de l'espace $E_{1}=R^{p}$ des $p$ variables sur le sous-espace $E_{10}=R^{l}$ correspondant à un sous-ensemble de $l$ variables $(l \leqq p)$ et de l'espace

( $\left.{ }^{3}\right)$ Si les colonnes de $C$ sont les vecteurs propres de $\dot{V}_{11}$ on a

$$
X=C Y \text {, }
$$

comme $C C^{t}=C^{t} C=I$, il en résulte que

$$
Y=C^{t} X
$$

vol. $14, \mathrm{n}^{\circ} 1$, février 1980 
$E_{2}=R^{p}$ des $p$ facteurs sur un sous-espace $E_{20}=R^{k}$ correspondant à un sousensemble de $k$ facteurs $(k \leqq p)$. Pour cela nous disposons de :

$B_{1}$ : Matrice de projection de $R^{p}$ sur $R^{l}\left(B_{1}\right.$ sera une matrice diagonale dont une sous-matrice de rang $l$ est une matrice unité et dont les autres éléments sont nuls).

$B_{2}$ : Matrice de projection de $R^{p}$ sur $R^{k}\left(B_{2}\right.$ est construit selon le même principe que $B_{1}$ ).

A l'aide de $B_{1}$ et $B_{2}\left({ }^{4}\right)$, nous pouvons compléter le schéma de dualité du paragraphe $\mathrm{V}$ :

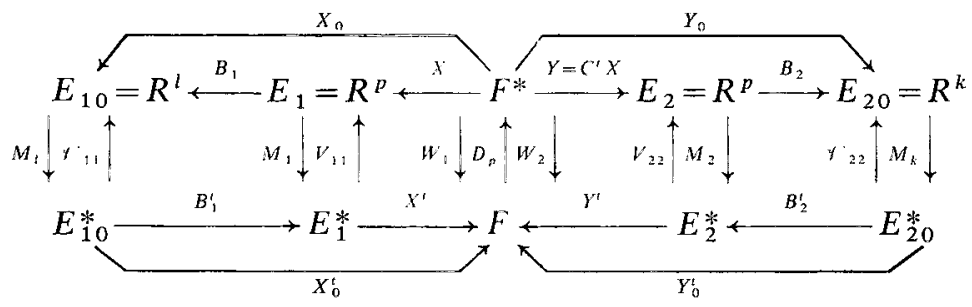

Dans ce schéma remarquons que :

$$
\begin{gathered}
\mathscr{V}_{11}=X_{0} D_{p} X_{0}^{t}=B_{1} X D_{p} X^{t} B_{1}^{t}=B_{1} V_{11} B_{1}^{t}, \\
\mathscr{V}_{22}=Y_{0} D_{p} Y_{0}^{t}=B_{2} Y D_{p} Y^{t} B_{2}^{t}=B_{2} C^{t} V_{11} C B_{2}^{t}=B_{2} D_{\lambda} B_{2}^{t}, \\
\mathscr{V}_{12}=X_{0} D_{p} Y_{0}^{t}=B_{1} X D_{p} X^{t} B_{2}^{t}=B_{1} V_{11} C B_{2}^{t}, \\
\mathscr{V}_{21}=B_{2} C^{t} V_{11} B_{1}^{t} .
\end{gathered}
$$

Nous pouvons de même qu'au paragraphe IV définir $\mathscr{U}_{1}$ et $\mathscr{U}_{2}$ comme suit

$$
\text { si }\left\{\begin{array} { l } 
{ \mathscr { W } _ { 1 } = X _ { 0 } ^ { t } M _ { l } X _ { 0 } } \\
{ \mathscr { W } _ { 2 } = Y _ { 0 } ^ { t } M _ { k } Y _ { 0 } }
\end{array} \text { alors } \left\{\begin{array}{l}
\mathscr{U}_{1}=\mathscr{W}_{1} D_{p}, \\
\mathscr{U}_{2}=\mathscr{W}_{2} D_{p}
\end{array}\right.\right.
$$

et poser le produit scalaire

$$
P\left(\mathscr{U}_{1}, \mathscr{U}_{2}\right)=\operatorname{Trace}\left(\mathscr{V}_{21} M_{1} \mathscr{V}_{12} M_{k}\right) .
$$

$D$ 'après les égalités précédentes, on en déduit que

$$
P\left(\mathscr{U}_{1}, \mathscr{U}_{2}\right)=\text { Trace }\left(B_{2} C^{t} V_{11} B_{1}^{t} M_{l} B_{1} V_{11} C B_{2}^{t} M_{k}\right) \text {. }
$$

Or $V_{11} C=C D_{\lambda}$ de même $C^{t} V_{11}=D_{\lambda} C^{t}$.

Donc

$$
P\left(\mathscr{U}_{1}, \mathscr{U}_{2}\right)=\operatorname{Trace}\left(B_{2} D_{\lambda} C^{t} B_{1}^{t} M_{l} B_{1} C D_{\lambda} B_{2}^{t} M_{k}\right)
$$

$\left({ }^{4}\right)$ Les notations sont les mêmes entre une application et la matrice qui lui est associée. 
Si on pose $D_{\lambda k}$ la matrice déduite de $D_{\lambda}$ dans laquelle on a annulé les $p-k$ éléments ne correspondant pas aux facteurs choisis et $A$ la matrice déduite de la matrice $C^{t}$ dans laquelle on a annulé les $p-k$ lignes ne correspondant pas aux facteurs choisis et les $p-l$ colonnes ne correspondant pas aux variables choisies, on peut vérifier que

$$
B_{2} D_{\lambda} C^{t} B_{1}^{t}=D_{\lambda k} A
$$

et que

$$
B_{1} C D_{\lambda} B_{2}^{t}=A^{t} D_{\lambda k} .
$$

On en déduit la formulation suivante du produit scalaire

$$
P\left(\mathscr{U}_{1}, \mathscr{U}_{2}\right)=\operatorname{Trace}\left(D_{\lambda k} A M_{1} A^{t} D_{\lambda k} M_{k}\right) \text {. }
$$

\section{EXPRESSION DE LA « CONTRIBUTION » EN FONCTION DU PRODUIT SCALAIRE ENTRE OPÉRATEURS}

Avant de tenter de faire le lien entre la «contribution 》 $\mathscr{C}_{\mathrm{oy}_{\mathrm{k}} / x_{l}}$ et le produit scalaire $P\left(\mathscr{U}_{1}, \mathscr{U}_{2}\right)$, il faut faire les remarques suivantes :

$1^{\circ} \mathscr{Y}_{k}$ et $\mathscr{X}_{l}$ étant des vecteurs aléatoires, nous devons passer à des réalisations de ces vecteurs : soit $Y_{k}$ et $X_{l}$;

$2^{\circ}$ si on a une matrice quelconque $T_{1}$ à $k$ lignes et $k$ colonnes qui est une sous-matrice d'une matrice $T_{2}$ dans laquelle tous les autres éléments autres que ceux de $T_{1}$ sont nuls alors la trace de ces deux matrices reste inchangée : Trace $\left(T_{1}\right)=\operatorname{trace}\left(T_{2}\right)$. Nous pouvons donc confondre ces deux types de matrice au niveau de leur trace.

Ceci étant posé, si nous prenons pour métriques $M_{k}$ et $M_{l}$ les métriques suivantes:

$M_{k}=D_{\lambda k}^{-1}$ (soit la matrice des inverses des valeurs propres) et $M_{l}=I$ (matrice unité), alors :

$$
P\left(\varkappa_{1}, \varkappa_{2}\right)=\text { Trace }\left(D_{\lambda k} A A^{t}\right)
$$

soit :

$$
P\left(\mathscr{U}_{1}, \mathscr{U}_{2}\right)=\text { Trace }\left(A^{t} D_{\lambda k} A\right)
$$

D'autre part, exprimons $\mathscr{U}_{1}$ :

$$
\mathscr{U}_{1}=\mathscr{W}_{1} D_{p}=X_{0}^{t} M_{l} X_{0} D_{p}=X^{t} B_{1}^{t} M_{l} B_{1} X D_{p}
$$

Calculons la trace de $\mathscr{U}_{1}$ avec l'hypothèse $M_{l}=I$ :

Trace $\left(\mathscr{U}_{1}\right)=\operatorname{Trace}\left(X^{t} B_{1}^{t} B_{1} X D_{p}\right)=\operatorname{Trace}\left(B_{1} X D_{p} X^{t} B_{1}^{t}\right)$

$$
=\operatorname{Trace}\left(B_{1} V_{11} B_{1}^{t}\right)=\operatorname{Trace}\left(B_{1} C D_{\lambda} C^{t} B_{1}^{t}\right) \text {. }
$$

vol. $14, \mathrm{n}^{\circ} 1$, février 1980 
Si nous remarquons que le produit $C^{t} B_{1}^{t}$ n'est autre qu'une matrice qui contient la matrice $B$ du paragraphe II et des éléments nuls partout ailleurs, nous pouvons écrire :

$$
\text { Trace }\left(\mathscr{U}_{1}\right)=\operatorname{Trace}\left(B^{t} D_{\lambda} B\right) .
$$

A ce point, nous disposons de tous les éléments pour exprimer $\mathscr{C}_{Y_{k} / X_{t}}$ en fonction des opérateurs $\mathscr{U}_{1}$ et $\mathscr{U}_{2}$ :

$$
\mathscr{C}_{Y_{k} / X_{t}}=\frac{\operatorname{Trace}\left(\mathscr{U}_{1} \mathscr{U}_{2}\right)}{\operatorname{Trace}\left(\mathscr{U}_{1}\right)} \quad \text { soit } \quad \mathscr{C}_{Y_{k} / X_{t}}=\frac{P\left(\mathscr{U}_{1}, \mathscr{U}_{2}\right)}{\operatorname{Trace}\left(\mathscr{U}_{1}\right)} .
$$

\section{COMMENTAIRES}

On pourrait penser pouvoir établir la mesure symétrique de $\mathscr{C}_{\mathscr{Y}_{k} / \mathscr{X}_{2}}$ soit $\mathscr{C}_{\mathscr{X}_{1} / \mathscr{G}_{k}}$. Nous ne détaillerons pas les calculs, mais si nous suivons la démarche du paragraphe II, nous obtenons l'expression de $\mathscr{C}_{\mathscr{X}_{1} / \mathscr{y}_{k}}$ sous la forme

$$
\mathscr{C}_{\mathscr{X}_{1} / \mathscr{Q}_{k}}=\frac{\sum_{i=1}^{k} \sum_{j=1}^{l} c_{i j}^{2} \operatorname{var}\left(X_{j}\right)}{\text { Trace } D_{\lambda k}}+\frac{T R}{\text { Trace } D_{\lambda k}} .
$$

Dans cette expression $T R$ désigne les termes rectangles dus au fait que $E\left(\mathscr{X} \mathscr{X}^{t}\right)$ n'est pas une matrice diagonale.

Si nous nous limitons comme mesure de la contribution cherchée à la quantité

$$
\mathscr{C}_{\mathscr{X}_{1} / \mathscr{Y}_{k}}=\frac{\sum_{i=1}^{k} \sum_{j=1}^{l} c_{i j}^{2} \operatorname{var}\left(X_{j}\right)}{\operatorname{Trace} D_{\lambda_{k}}},
$$

en négligeant les termes rectangles (pensant avoir là une sorte de contribution " pure » des variances des variables), on se heurte à des paradoxes : en effet, sous l'hypothèse de variables réduites, si l'on pose $k=1$ et $l=n$ on obtient la quantité

$$
\mathscr{C}_{\mathscr{X}_{1} / \mathscr{Y}_{k}}=\frac{\sum_{j=1}^{n} c_{i j}^{2}}{\lambda_{k}}=\frac{1}{\lambda_{k}},
$$

qui peut, s'il s'agit des premiers facteurs pour lesquels $\lambda_{k} \geqq 1$, être supérieur à l'unité; ce qui voudrait dire que la contribution de $\mathscr{X}_{l}$ à la variance de $\mathscr{Y}_{k}$ est supérieure à $100 \%$ ! L'explication du phénomène est simple : TR peut être négatif. 
Nous devons donc nous contenter de la mesure $\mathscr{C}_{\mathscr{Y}_{\mathrm{k}} / \mathscr{X}_{1}}$ qui, si elle présente des limites dues à la remarque précédente (qui montre que ce n'est pas une distance), a toutefois le gros intérêt d'être très suggestive. On peut penser à deux types d'applications :

- interprétation des résultats d'une analyse en composantes principales;

- validation de procédures telles que "l'échantillonnage dans une population de variables aléatoires réelles " [4] ou telles que la recherche de " séries chronologiques multiples résumées" [2].

On peut toutefois se ramener à un concept de distance au prix d'une certaine complexification (voir [6]).

\section{BIBLIOGRAPHIE}

1. A. Bonnafous, La logique de l'investigation économétrique, Dunod, Paris, 1973.

2. J.-M. BraUn, Étude des séries chronologiques multiples par l'Analyse des Données, Rapport C.E.A.-R-4561, 1974.

3. F. CAillez et P. PAges, Introduction à l'Analyse des Données, S.M.A.S.H., Paris, 1976.

4. Y. ESCOUFIER, Échantillonnage dans une population de variables aléatoires réelles, Thèse de doctorat d'État, Université de Montpellier, 1970.

5. J. P. Pages, Y. Escoufier et P. CAzes, Opérateurs et analyse des tableaux à plus de 2 dimensions, Cahier $\mathrm{n}^{\circ} 25$ du Bureau universitaire de recherche opérationnelle, Institut de Statistiques, Paris, 1976.

6. S. SELlam, Un concept de proximité entre sous-espaces vectoriels et son application à l'Analyse des Données. Thèse $3^{\mathrm{C}}$ Cycle, Université Lyon-I, 1979. 